\title{
Modeling Of Power Line Communication Channel For Automatic Meter Reading System With LDPC Codes
}

\author{
B. Rajkumarsingh and Namratha S. Poonye \\ Dept. of Electrical and Electronic Engineering \\ University of Mauritius \\ Reduit, Mauritius \\ Email: b.rajkumarsingh@uom.ac.mu / namratha.swapna@gmail.com
}

\begin{abstract}
In this era of modernization, one of the promising emerging technologies is Power Line Communication (PLC) system. In previous research fields, modeling of PLC channel, mostly for indoor applications has been studied. However, the need to study that for outdoor applications, such as the Automatic Meter Reading (AMR) systems is also vital. Moreover, standardization bodies have considered the use of LDPC codes restricted for indoor systems. Thus, in this paper, not only we model the PLC channel based on AMR applications, but also, we apply LDPC coding scheme to the system. To accomplish the objectives, firstly, we model the PLC-AMR channel, which includes multipath phenomenon. Additionally, PLC noise, usually occurring in the channel, is modeled. The modulation technique applied is BPSK and the performance of the system with varying load impedances is compared. The coded system consists of irregular LDPC codes, with two different constructions of the Parity-Check matrix, namely that by Radford Neal and reduced size of DVBS2. The performances of respective systems are then compared. Using LDPC by Radford Neal, the performances are analyzed with varied code rates.
\end{abstract}

Keywords-Power Line Communication; Automatic meter reading; multipath channel; LDPC codes

\section{INTRODUCTION}

Power Line Communication, on a general aspect simply means to transmit communication signals through the already built power distribution network. The use of the power lines as a means of communication media has always been part of the communication network but has remained under-developed. Its evolution to a bi-directional system occurred in the 90's and was used in smart grid applications. The new millennium has led to the advent of the PLC technology which has migrated to broadband applications for internet access [1].

Development in the PLC technology includes both indoor and outdoor applications. In indoor applications, such as home automation and internet access, users benefit from a combined scheme of PLC: accessing data from the same electrical sockets which supply power to their appliances. With regard to outdoor applications, such as Automatic Meter Reading (AMR), this provides a real-time data access anytime by the utility company or the consumer himself, thus providing a more practical and efficient means of meter reading. The device also provides additional features such as secluded monitoring and feedback on the amount of power consumed, power-cut notification and remote detection of suspicious electricity use [2]. PLC-AMR systems are regarded as the most cost efficient communication means since the global infrastructure of the system has already been built.

Standardization bodies have been set up to have an eye-watch on the spectrum and to regularize the system. The standards in power-line technologies are mostly based on the range of frequency used by devices namely, the Ultra Narrowband PLC, UNB-PLC (0.3 to $3 \mathrm{kHz}$ ), Narrowband PLC, NB-PLC (3 to 500 $\mathrm{kHz}$ ), Broadband PLC, BB-PLC (as from 1.8 MHz) [3], [4]. In comparison with UNB-PLC system, which has a limited data rate, the NB-PLC systems are regarded to be more efficient. Most NB-PLC devices implement IEEE 1901.2, ITU.T G.9904 and ITU.T G.9903 standards whereas BB-PLC systems generally use the IEEE 1901.2010, ITU-T G.hn standards [5] and most recently, the IEEE 1905.1 standard, which merges different means of communication media [6]. The European standard, CENELEC defined by EN 50065 regularizes PLCAMR applications in a frequency range of 3-95 kHz, named as band A [7].

As the data signal travels from the transmitter to the receiver, it encounters several constraints: attenuation, dependence on distance of propagation, multipath effect and various sources of interfering signals [1]. The main challenge till now, has been to go through the modeling of these weaknesses, such that the performance of the overall system can be analyzed. Thus, section II illustrates the most commonly used modeling technique of the PLC channel, together with the modeling approaches of noise in PLC [8], [9]. However, these previous research works have been concentrating on the modeling of the PLC multipath channel for indoor applications. Section III, on the contrary, will focus on the evaluation of PLC-AMR multipath channel parameters. This differs from the indoor applications, based on the type of cables used in the low voltage electrical network topology. Thus, new parameters, which characterize the PLC-AMR channel, are evaluated. Furthermore, for complete modeling of the channel, it is vital to analyze the attenuation effect for that particular application. From the overview on previous research in the field, it can be deduced 
that the PLC channel is dependent on its connected load impedance. Thus, another major aspect is the analysis of performances with varying load impedances on the PLC-AMR system. Furthermore, improving a communication system, using an appropriate channel encoder is a common scheme. This further inspires the aim of the thesis by selecting a powerful Forward Error Correction FEC method: the LDPC codes. Section IV illustrates the modeling of the complete PLC-AMR system and section $\mathrm{V}$ provides various analysis performed on that system. Section VI then concludes the paper based on the results obtained.

\section{LITERATURE REVIEW}

\section{A. Multipath PLC channel}

An electrical network topology consists of several branched networks. With point of impedance discontinuities, the main transmitted signal will not have a direct path to the receiver, instead, several reflections of the wave occur before reaching its destination. This results in echoes of the transmitted signal [10].

The paper work of [8] explains the multipath effect by using a T-network topology whereby only one-branched network is considered (Fig. 1 refers). The structure is divided into three segments, namely (1), (2), and (3) each with their respective characteristic impedances, $\mathrm{Z}_{01}, \mathrm{Z}_{02}$ and $\mathrm{Z}_{03}$, Load impedances $Z_{A}, Z_{C}$ and $Z_{D}$ and lengths $1_{1}, l_{2}$ and $l_{3}$. Assuming matched impedances on both segment (1) and (3), reflections will only occur on the branch $\mathrm{BC}$, since $\mathrm{Z}_{\mathrm{C}} \neq \mathrm{Z}_{02}$. Hence, Node $\mathrm{B}$ is referred to as the point of mismatch. By taking into account these assumptions, the reflection and transmission factors present on the network are as follows: $\mathrm{r}_{\mathrm{BA}}, \mathrm{r}_{\mathrm{BC}}, \mathrm{r}_{\mathrm{CB}}, \mathrm{t}_{\mathrm{AB}}$ and $\mathrm{t}_{\mathrm{CB}}$.

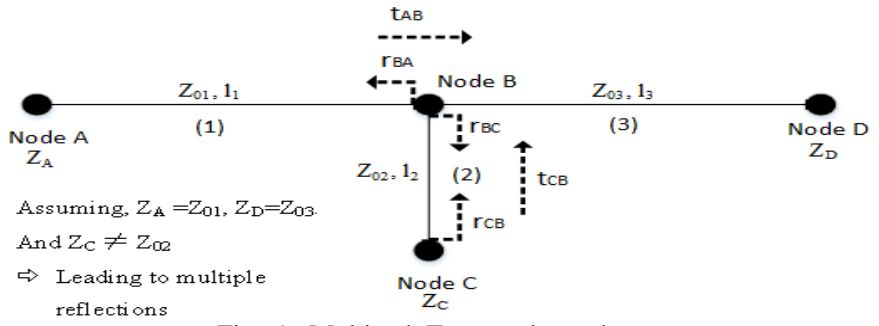

Fig. 1. Multipath T-network topology

The followings are used to evaluate the reflection and transmission factors:

$$
\begin{aligned}
& \mathrm{r}_{\mathrm{BA}}=\frac{Z_{\mathrm{zm}}-Z_{\mathrm{on}}}{Z_{\mathrm{zm}}+Z_{\mathrm{gn}}} \quad ; \mathrm{Z}_{23}=\mathrm{Z}_{02} \| \mathrm{Z}_{03} \\
& \mathrm{r}_{\mathrm{BC}}=\frac{z_{21}-z_{02}}{z_{2 \mathrm{xz}}+z_{0 z}} \quad ; Z_{13}=Z_{01} \| Z_{03} \\
& \mathrm{r}_{\mathrm{CB}}=\frac{z_{C}-z_{0 a}}{z_{C}+z_{03}} \\
& \mathrm{t}_{\mathrm{AB}}=1+\mathrm{r}_{\mathrm{BA}} \\
& \mathrm{t}_{\mathrm{CB}}=1+\mathrm{r}_{\mathrm{BC}}
\end{aligned}
$$

The transfer function of the channel encompasses three types of attenuation which are due to the multiple reflections, dielectric and conductor loss and the delay in the transmitted signal [9]. Thus, the transfer function $H(f)$ of the PLC channel can be expressed as:

$$
H(f)=\sum_{i=0}^{W} g_{i} \cdot A\left(f_{i} d_{i}\right) \cdot e^{-j z-f x_{i}}
$$

$N$ is the total number of propagation paths due to multiple reflections. $g_{\mathrm{i}}$ is the attenuation factor representing the weight of the $i^{\text {th }}$ path, generally complex and frequency dependent. $A\left(f_{0}, d_{i}\right)$ is the attenuation caused by loss in cables, which is dependent on the total length of the path $d_{i}$ and frequency of the propagating wave $f$. $\tau_{\mathrm{i}}$ is the delay corresponding to the particular path length, $d_{i}$.

Referring to Fig. 1, the table below gives a generalized case illustrating the possible propagation paths and the evaluation of the weighting factor $\theta_{\mathrm{i}}$.

TABLE I. SIGNAL PATH PROPAGATIONS

\begin{tabular}{|c|c|c|c|}
\hline $\begin{array}{c}\text { Path } \\
\text { number, } \boldsymbol{i}\end{array}$ & Wave path & $\begin{array}{c}\text { Weighting } \\
\text { factor } \boldsymbol{g}_{\boldsymbol{i}}\end{array}$ & $\begin{array}{c}\text { Path length, } \\
\boldsymbol{d}_{\boldsymbol{i}}\end{array}$ \\
\hline 1 & $\mathrm{ABD}$ & $\mathrm{t}_{\mathrm{AB}}$ & $1_{1}+1_{3}$ \\
\hline 2 & $\mathrm{ABCBD}$ & $\mathrm{t}_{\mathrm{AB}} \cdot \mathrm{r}_{\mathrm{CB}} \cdot \mathrm{t}_{\mathrm{CB}}$ & $1_{1}+2 \mathrm{l}_{2}+\mathrm{l}_{3}$ \\
\hline 3 & $\mathrm{ABCBCBD}$ & $\begin{array}{c}\mathrm{t}_{\mathrm{AB}} \cdot \mathrm{r}_{\mathrm{CB}}{ }^{2} \cdot \\
\mathrm{r}_{\mathrm{BC}} \cdot \mathrm{t}_{\mathrm{CB}}\end{array}$ & $1_{1}+41_{2}+1_{3}$ \\
\hline $\mathrm{N}$ & $\mathrm{AB}(\mathrm{CB})^{\mathrm{N}-1} \mathrm{D}$ & $\begin{array}{c}\mathrm{t}_{\mathrm{AB}} \cdot \mathrm{r}_{\mathrm{CB}} \cdot \\
\left(\mathrm{r}_{\mathrm{BC}} \cdot \mathrm{r}_{\mathrm{CB}}\right)^{\mathrm{N}-2} \\
\cdot \mathrm{t}_{\mathrm{CB}}\end{array}$ & $1_{1}+2(\mathrm{~N}-1) 1_{2}+1_{3}$ \\
\hline
\end{tabular}

The delay $\tau_{\mathrm{i}}$ can be calculated by using the following equation,

$$
\tau_{i}=\frac{d_{i} \sqrt{\varepsilon_{s}}}{c_{0}}
$$

Where, $c_{0}$ is the speed of light, $\varepsilon_{\mathrm{r}}$ is the relative permittivity of dielectric medium.

The attenuation factor due to the cable losses can be calculated using (8).

$$
A\left(f, d_{i}\right)=e^{-a(f)} d_{1}
$$

Using (9), $\alpha(f)$ can determined. This includes both losses: the conductor and dielectric loss [11].

$$
\alpha(f)=\frac{R}{2 \cdot Z_{0}}+\frac{G \cdot Z_{g}}{2}
$$

\section{B. PLC noise}

The extensive analysis and measurement carried out by [12], [13] have shown that the PLC channel consists of four types of PLC noise, listed as follows: Colored Background Noise, Narrowband Noise, Synchronous Periodic Impulsive Noise, Asynchronous Periodic Impulsive Noise [11], [14]. From [15], it has been shown that the first two types of noise can be lumped 
into Background Noise and that the last two can be categorized as Impulsive Noise.

Background noise is often modelled in the frequency domain which is based on measurement of noise frequency spectrum. One of the proposed methods for its modeling is the inclusion of specific Power Density Functions (PDFs) in a decreasing modelled spectrum. Thus, the statistical characteristics of the noise at a given frequency can be fully described. Methods of modeling are sum of two Rayleigh distributions, the log-normal distribution or the Gaussian distribution [15].

Impulsive noise can be modelled in both frequency [16] and time domain [17]. In the time domain analysis, one approach used to model the Impulsive noise, is to consider the set of impulses as a pulse train. That is, the impulses are close enough to be categorized into their corresponding impulse envelopes, as illustrated in Fig. 1 in [17]. The pulse train are characterized by the following parameters: the pulse amplitude $A_{i}$, the pulse width $T_{w, i}$ and the inter-arrival time $T_{\text {iat, } i}$ of the $i^{\text {th }}$ impulse envelope. From measurement of real-valued noise waveforms, the statistical properties of these parameters have been determined in [13]. A summary of these properties is given in TABLE II.

TABLE II. MEASURED PARAMETERS OF IMPULSIVE NOISE

\begin{tabular}{|c|c|c|}
\hline Parameters & $\%$ of parameters & Typical values \\
\hline \multirow{2}{*}{ Amplitudes } & $90 \%$ & Range of $100-200 \mathrm{mV}$ \\
\hline & $<1 \%$ & $>2 \mathrm{~V}$ \\
\hline \multirow{2}{*}{ Pulse widths } & $1 \%$ & $>500 \mu \mathrm{s}$ \\
\hline & $0.2 \%$ & $>1 \mathrm{~ms}$ \\
\hline \multirow[b]{2}{*}{$\begin{array}{c}\text { Inter-arrival } \\
\text { times }\end{array}$} & $90 \%$ & $<200 \mathrm{~ms}$ \\
\hline & $\begin{array}{c}30 \% \text { (representing } \\
\text { the third types of } \\
\text { noise) }\end{array}$ & 10 or $20 \mathrm{~ms}$ \\
\hline
\end{tabular}

\section{EVALUATION OF PLC-AMR CHANNEL PARAMETERS}

The PLC-AMR devices allow every user's meter to connect to their respective modem. The PLC signal is then transmitted from the end-consumer to the data concentrator found at the lowvoltage side of the distribution transformer. In [18] it has been stated that distribution transformers will severely attenuate the communication signal (around 40-60 dB). Thus, the signal will be only limited from one transformer distributing power to its connected houses.

The approach used to model the PLC channel is depicted from the basic theory of two-wire transmission line theory [19] and the background theory described in section II, A. The principles are then applied to the described AMR system.

The proposed $\mathrm{T}$ network topology to be used in PLC-AMR system is shown in Fig. 2. It illustrates the main supply cable from the transformer to the mismatch point (B) and the two house supply cables, connected from B to the two houses. It is assumed that the load impedances of House A and Transformer
$\mathrm{D}$ remain constant, i.e. $\mathrm{Z}_{\mathrm{A}}=\mathrm{Z}_{01}$ and $\mathrm{Z}_{\mathrm{D}}=\mathrm{Z}_{03}$. Thus, only the load impedance of House $C, Z_{C}$ varies. Also, since house supply cables are the same, $Z_{01}=Z_{02}$. The diagram also indicates the nominal cross section area, CSA $\left[\mathrm{mm}^{2}\right]$ of the wires.

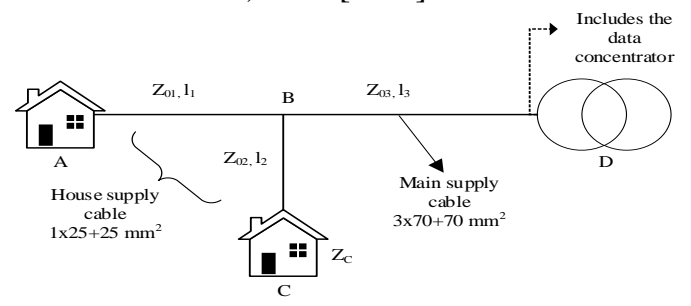

Fig. 2. Per phase proposed T-network distribution topology

The RLGC parameters are evaluated from (10) to (13) [19]. Due to the negligible phases of the characteristic impedances of the house and main supply cable, the formers are evaluated using (14). The evaluated line parameters, at a carrier frequency $f$ of $95 \mathrm{kHz}$, are given in TABLE III.

$$
\begin{aligned}
& \mathrm{R}=\frac{1}{3.20,4,5} \\
& \mathrm{~L}=\frac{\mathrm{E}}{\mathrm{B}} a \cosh \frac{\mathrm{D}}{\mathrm{z} a} \\
& \mathrm{G}=\frac{\operatorname{manh} x^{\prime \prime}}{\mathrm{x} \operatorname{sesh} \frac{\mathrm{a}}{\mathrm{x} x}} \\
& \mathrm{C}=\frac{\operatorname{mex}}{x \cosh \frac{5}{x x}} \\
& z_{0}=\sqrt{\frac{L}{C}}
\end{aligned}
$$

Where,

$\mathrm{R}=$ series resistance, per unit length $(\Omega / \mathrm{m})$.

$\mathrm{L}=$ series inductance, per unit length $(\mathrm{H} / \mathrm{m})$.

$\mathrm{G}=$ shunt conductance, per unit length $(\mathrm{S} / \mathrm{m})$.

$\mathrm{C}=$ shunt capacitance, per unit length $(\mathrm{F} / \mathrm{m})$.

$\mathrm{a}=$ radius of conductor $(\mathrm{m})$.

$\mathrm{D}=$ separating distance between center of wires $(\mathrm{m})$.

$\sigma_{\mathrm{c}}=$ conductivity of conductor $(\mathrm{S} / \mathrm{m})$.

$\delta_{\mathrm{c}}=\operatorname{skin} \operatorname{depth}(\mathrm{m})=1 /\left(\mathrm{f} \mu \pi \sigma_{\mathrm{c}}\right)$

$\omega=$ angular frequency $(\mathrm{rad} / \mathrm{s})=2 \pi \mathrm{f}$.

\begin{tabular}{|c|c|c|c|c|c|}
\hline \multicolumn{6}{|c|}{$\begin{array}{l}\text { TABLE III. EVALUATED LINE PARAMETERS } \\
\qquad \begin{array}{c}\text { Primary line parameters } \\
\end{array}\end{array}$} \\
\hline $\mathrm{CSA}\left(\mathrm{mm}^{2}\right)$ & $\begin{array}{c}R \\
(\Omega / m)\end{array}$ & $L(\mu H / m)$ & $\begin{array}{c}G \\
(\mu S / m)\end{array}$ & $\begin{array}{c}C \\
(n F / m)\end{array}$ & $Z_{0}(\Omega)$ \\
\hline$(1 \times 25)+2$ & $\begin{array}{r}4.4 \\
\times 10^{-2}\end{array}$ & 0.250 & 0.640 & 0.107 & 48.2 \\
\hline$(1 \times 70)+7$ & $\begin{array}{r}2.2 \\
\times 10^{-2}\end{array}$ & 0.177 & 0.898 & 0.150 & 34.3 \\
\hline
\end{tabular}

$\mu=$ permeability of dielectric medium $(\mathrm{H} / \mathrm{m})$.

$\varepsilon_{0}=$ permittivity of free space $(\mathrm{F} / \mathrm{m})$.

$\varepsilon_{\mathrm{r}}=$ relative permittivity of dielectric medium.

$\varepsilon=$ permittivity of dielectric medium $(\mathrm{F} / \mathrm{m})=\varepsilon_{0} . \varepsilon_{\mathrm{r}}$. 
In an electrical distribution, connected loads have varying impedances, on which the weighting factors are dependent. Thus, for various load impedances $\left(Z_{C}=10,50\right.$ and $\left.100 \Omega\right)$, the trend in the factors is given in TABLE IV, together with the lengths of the signal paths $d_{i}$, which are based on a chosen length of segments. The trend in delay factors $\tau_{i}$ of the transmitted signal are also evaluated. The time taken for the transmitted signal to reach the receiver is in the order of $\mu \mathrm{s}$. It has been observed that for each increasing path length, the delay roughly increases by a factor of 2 .

TABLE IV. EVALUATED MULTIPATH PARAMETERS

\begin{tabular}{|c|c|c|c|c|c|c|c|}
\hline \multirow{7}{*}{$\begin{array}{c}\mathbf{Z}_{\mathbf{C}} \\
(\boldsymbol{\Omega})\end{array}$} & \multicolumn{7}{|c|}{ Path number, $\tilde{i}$} \\
\hline & 1 & 2 & 3 & & 4 & 5 & 6 \\
\hline & \multicolumn{7}{|c|}{ Path length $d_{i}(\mathrm{~m})$} \\
\hline & 210 & 350 & 490 & & 30 & 770 & 910 \\
\hline & \multicolumn{7}{|c|}{ Delay $\tau_{i}(\mu \mathrm{s})$} \\
\hline & 1.084 & 1.807 & 2.530 & & 253 & 3.976 & 4.699 \\
\hline & \multicolumn{7}{|c|}{ Weighting factor, $g_{i}$} \\
\hline 50 & 0.5877 & 0.0064 & $-486 \times 1$ & $3.70 \times 1$ & & $-2.82 \times$ & $2145 \times 11$ \\
\hline 10 & 0.5877 & -0.2267 & -0.0613 & -0.0166 & & -0.0045 & -0.0012 \\
\hline 100 & 0.5877 & 0.1208 & -0.0174 & 0.0025 & & -0.0004 & 0.0001 \\
\hline
\end{tabular}

Fig. 3 illustrates the attenuation profile based on the cables losses, for the each path length (same as in TABLE IV) on a frequency range up to $30 \mathrm{MHz}$. It can be observed that the PLC signal will face deeper attenuation as its traveling distance increases. At $10 \mathrm{MHz}$, the signal degrades by a $2.5 \mathrm{~dB}$ for every increasing $100 \mathrm{~m}$ length. Furthermore, the signal undergoes very high attenuation (greater than $-16 \mathrm{~dB}$ ) in the broadband region and negligible attenuation in the narrowband region. Since PLCAMR technologies operate in the CENELEC band $\mathrm{A}$, the attenuation due to cables losses is of $0 \mathrm{~dB}$. Moreover, from depicted (15), the attenuation at a particular frequency for a given path length can directly be obtained by analyzing the trend in the $k$ coefficient, which doubles in the order of $10^{-7}$, for every $\cong 100 \mathrm{~m}$ increase in path length, with $a_{0}$ approximately equals to 1 .

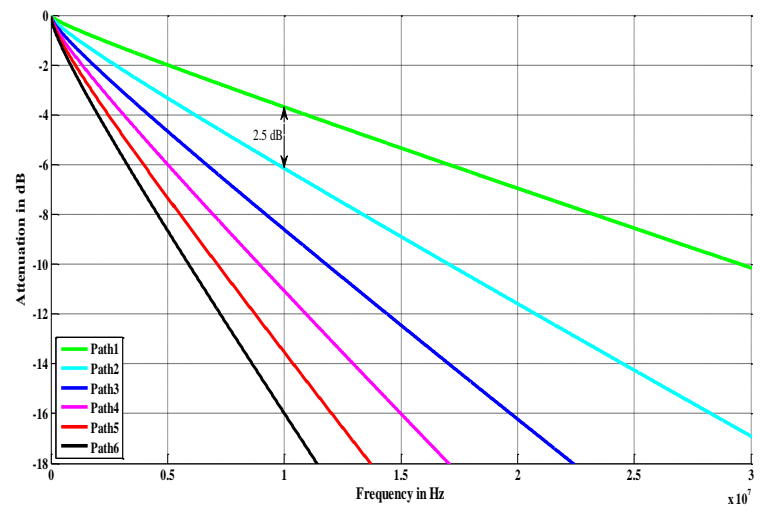

Fig. 3. Generated attenuation profile

$$
A\left(f, d_{i}\right)=a_{2} e^{-k, f}
$$

\section{COMPLETE SYSTEM MODEL}

Fig. 4 shows the connections of different blocks used to implement the PLC-AMR LDPC coded system. The overall system is realized using the simulator Simulink R2013a. The required parameters are evaluated in MATLAB R2013a, which are then transferred to their corresponding blocks.

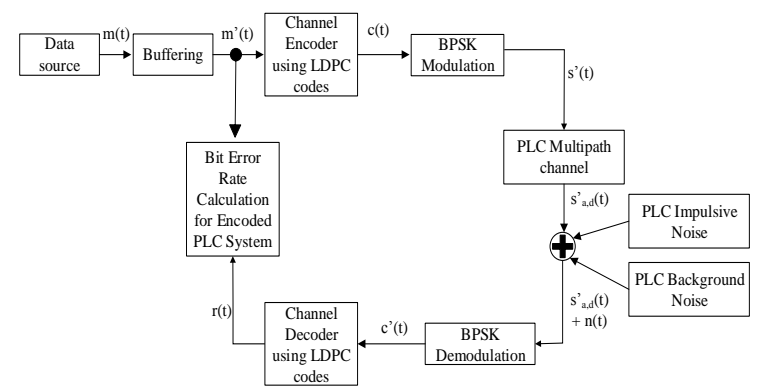

Fig. 4. Complete system model of LDPC coded PLC-AMR

Throughout the simulation, the data rate and packet size used are $500 \mathrm{kbps}$ and 800 , respectively [7]. The output of the data generator undergoes the buffering process, which in turn, sets its output length in accordance with the requirement of the input to the LDPC encoder. BPSK method is chosen as the modulation process [20]. A range of SNR is set for the evaluation of BER, with the signal power held at $0.5 \mathrm{~W}$.

\section{A. Modeling of PLC-AMR channel}

The PLC channel is realized by using a direct form FIR digital filter structure as illustrated in Fig 5, assuming that the main signal is synchronized. An FIR filter is usually characterized by an order of $\mathrm{N}$ with $\mathrm{N}+1$ numerator coefficients [9], [21]. Thus, taking into consideration the direct path of the signal, the total number of paths is $\mathrm{N}+1$. In this model, 6 signal propagation paths are chosen. The numerator coefficients denote the evaluated weighting factors. The delay for each signal path is assumed to be the duration of one output buffer frame, thus considering a worst-case scenario.

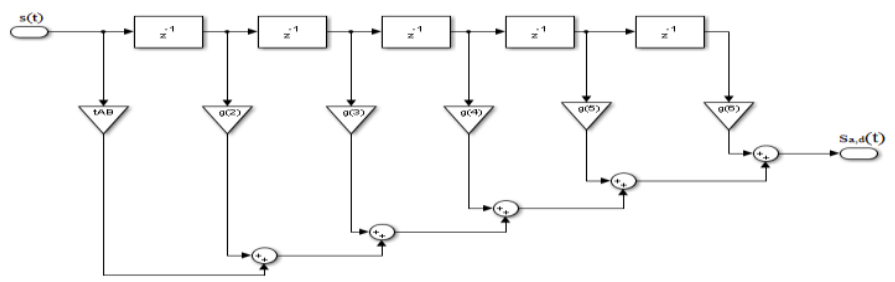

Fig. 5. Echo-based model in the form of an FIR filter

\section{B. Modeling of PLC noise}

Impulsive noise is modelled by generating a set of pulse trains with their corresponding parameters determined from the measured statistical characteristics. Assuming, periodicity in the 
pulse trains (Fig. 6), the total impulsive noise power $P_{\text {imp }}$ can be obtained as follows.

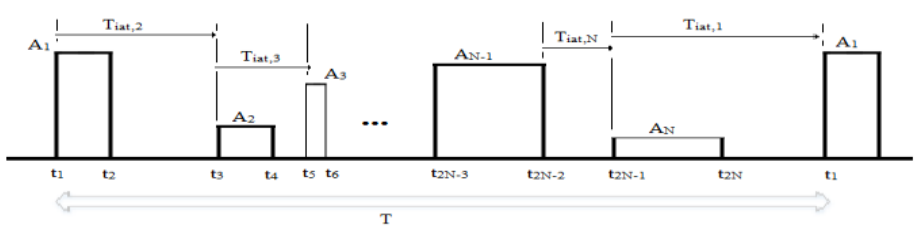

Fig. 6. Periodic pulse trains

The total period of $\mathrm{N}$ pulses, denoted here as $T$ is given by:

$T=\sum_{i=2}^{W} T_{i=E_{j}}$

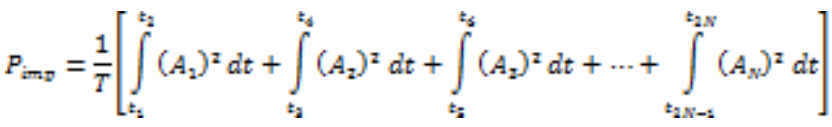

Since, $T_{z W}-T_{z W-1}=T_{\text {me }_{e} N}$

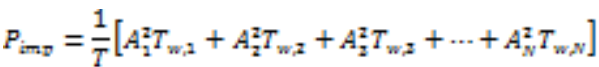

$$
=\frac{1}{T} \sum_{i=1}^{W} A_{i}^{z} T_{\mathrm{me} g}
$$

The sum of two Rayleigh noise generators, with appropriately set parameters, is used to model the background noise. From [22], the PDF for the modeling of Background noise using the sum of two Rayleigh distributions is given as follows:

$$
f(x)=a \frac{x}{\sigma_{1}} e^{\frac{x^{x}}{x z_{1}^{2}}}+(1-a) \frac{x}{\sigma_{2}} e^{\frac{x^{x}}{z z_{1}^{2}}}
$$

Where,

$a$ is the weighting factor with a typical value of 0.3 .

$\sigma_{1}{ }^{2}$ and $\sigma_{2}{ }^{2}$ are the variances representing the average power of the noise. Usually $\sigma_{1}^{2}=10 \sigma_{2}^{2}$.

From randomly chosen values of amplitude, pulse width and inter-arrival time, the total impulsive noise power is evaluated. For a set SNR, the total noise power is obtained, from which the background noise power is evaluated, thus obtaining the corresponding values of the variances.

\section{LDPC codes}

LDPC coding scheme is used to improve the PLC system's performance. The aim is to construct a $(n-k)$ by $n$ sparse parity-check matrix, where $k$ and $n$ are the lengths of message and codeword, respectively. The codeword length $n$ of the parity-check matrix is maintained fixed at 8100 . The approach used for the irregular LDPC construction is taken from [23], which is based on the simulation program used by Radford Neal. By varying $k$, the system's performance is analyzed with code rates of $1 / 2,1 / 4$ and $3 / 4$. As well, as a comparison, a parity-check matrix based on the DVB-S2 standards, with a code-word length reduced to 8100 of rate $1 / 2$ is used [24]. Both programs eliminate the girth 4 occurring in the matrix. The LDPC Decoder block is used to perform the decoding process by using the message passing technique, following the Sum-Product Algorithm (SPA), with each of its input element being the log-likelihood ratio LLR of the received bit.

\section{SiMULATION RESULTS}

\section{A. Performance of PLC-AMR system without LDPC codes}

The performance is examined by comparing four different scenarios without LDPC codes, presented in Fig 7. First is the 'ideal' case, whereby the system's performance is studied without the multipath channel. The last three performances are analyzed by varying the load impedances at 10,50 and $100 \Omega$. The performance of the PLC-AMR system is highly affected (by $5.5 \mathrm{~dB}$ ) in a multipath environment. Moreover, its performance is indebted to the evaluated weighting factors, which in turn depends on the discrepancy between the load impedance and characteristic impedance of the branched line on which reflection is occurring. It has been observed that the factors for the $10 \Omega$ case is highly destructive. This leads to a severe degradation in the performance (by $5.8 \mathrm{~dB}$ ) as compared to the case of $50 \Omega$, which involves less destructive factors. However, the significant improvement can only be observed as from an SNR of $8 \mathrm{~dB}$.

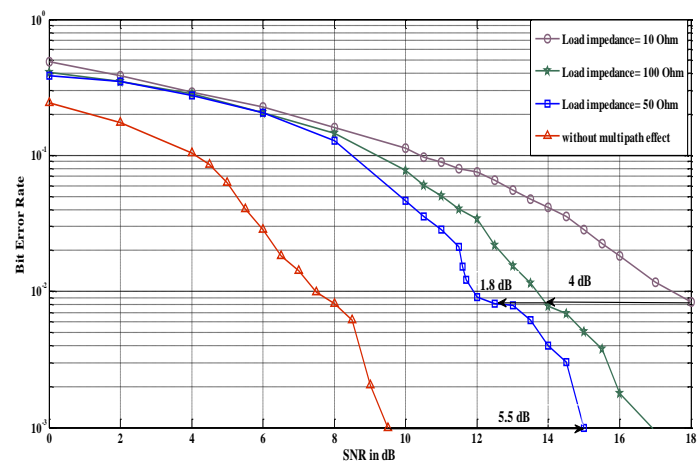

Fig. 7. Performance of PLC-AMR system with varied load impedances

\section{B. Performance of PLC-AMR system with LDPC codes by Radford Neal}

The improved trend of the $1 / 2$ rate LDPC codes by Radford Neal in the PLC-AMR system, from 10 to $100 \Omega$, reaching the $50 \Omega$ system, can be remarkably observed from Fig. 8. The performance of this system is significantly improved, by $6 \mathrm{~dB}$ for the $10 \Omega$ impedance, when compared with the no coding one. The coded system of $50 \Omega$ impedance, outperforms all the other varied impedances coded systems, with no error floor region. The coded performances reach the error floor region, whereby a 
rather slow improvement is encountered. Still, the performance of the $10 \Omega$ system tends to a more flattening curve, as compared to the other impedances in the floor region. The plausible reasons for that phenomenon may be due to low-weight near code-words or trapping sets in the parity-check matrix, which the message passing decoding algorithm cannot avoid [25], [26].

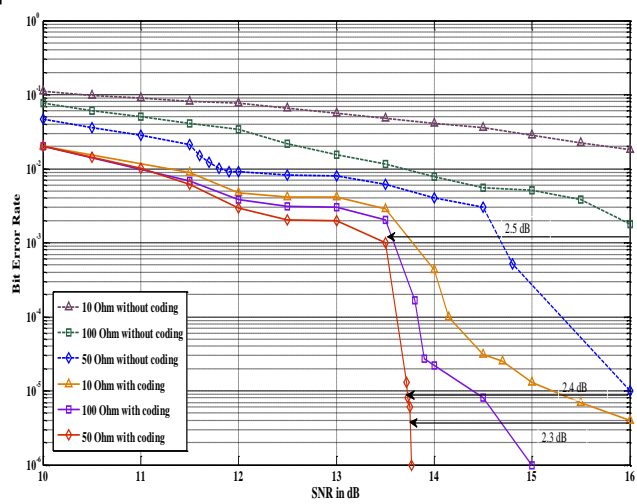

Fig. 8. Performance of LDPC coded PLC-AMR system of $1 / 2$ code rate with varied load impedances

\section{Performance comparison with reduced size LDPC codes of DVBS2}

Fig. 9 shows the comparative analysis between LDPC codes by Radford Neal and reduced size of DVBS2, applied in PLCAMR system of $10 \Omega$ impedance, both of rate $1 / 2$. It can be observed that no significant improvement occurs in the performance below $12.5 \mathrm{~dB}$ SNR. Nevertheless, beyond this range, in the waterfall region of coded LDPC of DVBS2, a relatively more abrupt decrease in BER is encountered. Furthermore, the performance with DVBS2 LDPC codes does not reach the error floor region, as compared to the other code. For the LDPC coded system by Radford Neal to reach the same performance to that of DVBS2, a $1.8 \mathrm{~dB}$ SNR is required.

During the construction of the LDPC codes, both programs eliminate the problem girth-4 problem. However, in [27], it has been stated that, even codes without girth 4, poor BER in high SNRs region can be encountered, due to the lower minimum weight in the parity-check matrix. The codes of DVBS2, however, eliminates that problem, thus outperforming the other code.

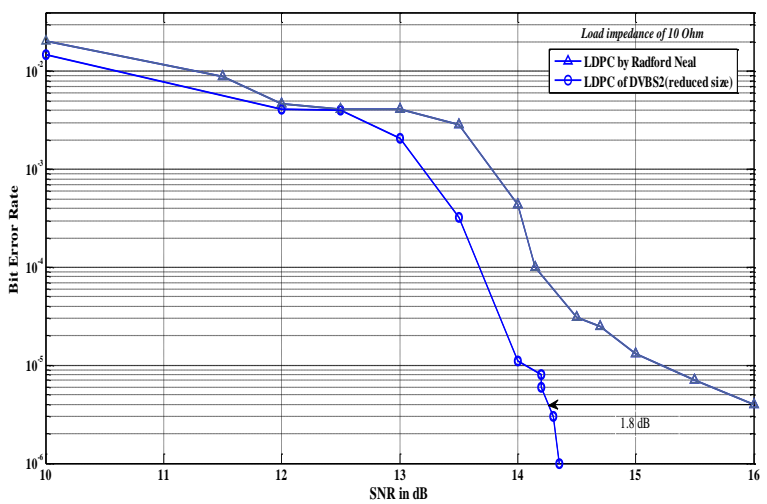

Fig. 9. Performance comparisons of LDPC codes by Radford Neal and of DVBS2

\section{Performance analysis with varying code-rates}

The comparison of performances with varying rates of $1 / 2,1 / 4$ and $3 / 4$ are illustrated in Fig. 10. The performance of the $1 / 4$ rate code encounters a significant improvement (lower BER as compared to the $1 / 2$ rate code) at high SNRs, at the expense of lower data transmission. Another visible aspect is that the $1 / 4$ rate code enters its waterfall region at a relatively smaller SNR of 13 $\mathrm{dB}$, as compared to the $1 / 2$ rate code. However, its performance quickly reaches its error floor region.

Moreover, the performance of the $3 / 4$ rate code is less desirable, relative to the other two rates. This degradation is expected due to an increase in the number of transmitted packets. Still, the performance of the LDPC $3 / 4$ rate code system is $3.2 \mathrm{~dB}$ SNR lower than the without coding system.

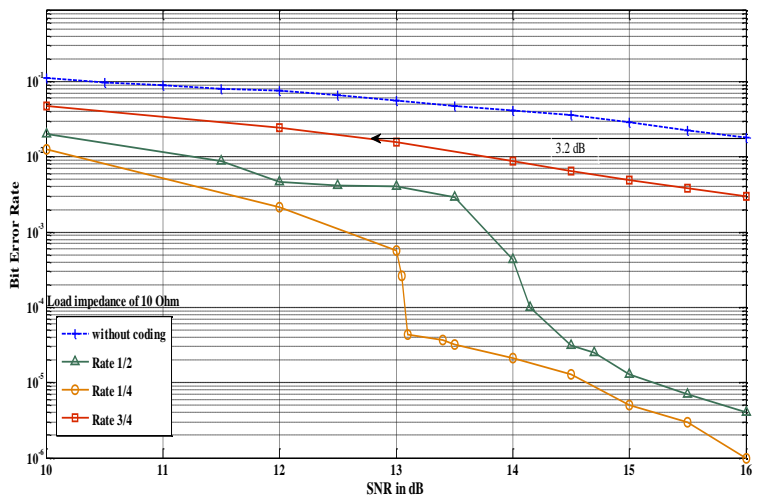

Fig. 10. Varied code rate performances using LDPC codes by Radford Neal

\section{E. Performance comparison with previous work}

Fig. 11 shows the performance comparison between the implemented system and that evaluated in [28]. The previous work analyses the performance of $1 / 2$ rate LDPC codes over a realistic PLC channel, using OFDM-BPSK scheme. Its severely 
retrograded performance (by $15 \mathrm{~dB}$ ) can clearly be observed, as compared to that simulated. This degradation in performance can be accounted by the use of regular LDPC codes (assumed to be worst-case scenario) with a lower codeword length of 4096.

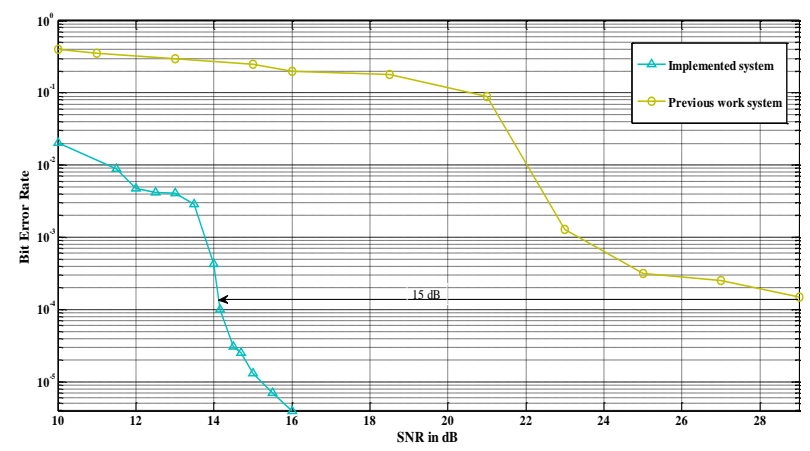

Fig. 11. Comparison of resulting performance with previous work

\section{CONCLUSION}

The analysis has shown an unpredictable nature of PLC-AMR channel, such that its performance cannot be as such anticipated for a specific load impedance, expect if the dependent factors of the weighting coefficients are estimated. As the deviation from the load impedance to the characteristic impedance of the cable, on which reflection is occurring, increases, the performance is expected to degrade.

Besides, the need to combat the multipath effect in this particular application revealed to be vital. Thus, the use of LPDC codes as a powerful FEC tool can definitely be implemented in revised standards for narrowband applications, instead of its restricted use to indoor technologies. It has been deduced that LDPC codes of reduced size DVBS2 provides a better performance that the codes by Radford Neal. Furthermore, as expected, irregular LDPC codes outperforms regular ones. Thus, the use of regular LDPC codes is not favored in implementation.

\section{REFERENCES}

[1] M. Yousuf and M. El-Shafei, "Power Line Communications: An Overview - Part I," in Proc. 4th International Conference on Innovations in Information Technology, Dubai, 2007, pp. 218 - 222.

[2] V. Bhashkar, S. Kumar, C. Prasad, G. Modi and R. Mahto, "A Reliable automatic meter reading system using power line distribution network," UARJ, vol. 1, no. 3,4, pp. $124-129,2012$.

[3] L. Berger, A. Schwager and J. Escudero-Garzás, "Power Line Communications for Smart Grid Applications," Hindawi Publishing Corporation, Journal of Electrical and Computer Engineering, vol. 2013, no. 712376, p. 16, 2012.

[4] M. Yousuf, S. Rizvi and M. El-Shafei, "Power Line Communications: An Overview - Part II," in Proc. 3rd International Conference on Information and Communication Technologies: From Theory to Applications, Damascus, 2008.
[5] CTI and UKA, "The OPEN meter Consortium," Description of current state-of-the-art of technology and protocols description of state-of-the-art of PLC-based access technology, 30 April 2009. [Online]. Available: http://www.openmeter.com/files/deliverables/OPEN-

Meter\%20WP2\%20D2.1\%20part2\%20v2.3.pdf. [Accessed 29 August 2013].

[6] IEEECS, "IEEE Standard for a Convergent Digital Home Network for Heterogeneous Technologies". U.S, IEEE Std 1905.1-2013, 2013 June 2013.

[7] IEEECS, "IEEE Standard for Low-Frequency (less than $500 \mathrm{kHz}$ ) Narrowband Power Line Communications for Smart Grid Applications". New York, IEEE Std 1901.2-2013, 31 October 2013

[8] M. Zimmermann and K. Dostert, "A Multipath Model for the Powerline Channel," IEEE Transactions on Communications, vol. 50, no. 4, pp. 553 $-559,2002$.

[9] H. Meng, et al., "A transmission line model for high-frequency power line communication channel," in Proc. International Conference on Power System Technology, 2002, pp. 1290 - 1295.

[10] L. T. Berger and G. Moreno-Rodriguez, "Power Line Communication Channel Modelling through Concatenated IIR-Filter Elements," JOURNAL OF COMMUNICATIONS, vol. 4, no. 1, pp. 41-51, 2009.

[11] Y. Gao, E. Liu, O. Bilal and O. T. Korhonen, "Channel modeling and modem design for broadband powerline communications," Presented in 7th International Symposium on Power-line Communications (ISPLC), Spain, 2004.

[12] M. Katayama, T. Yamazato and H. Okada, "A mathematical model of noise in narrowband power line communication systems," IEEE Journal on Selected Areas in Communications , vol. 24, no. 7, pp. 1267 - 1276 2006.

[13] M. Zimmermann and K. Dostert, "An Analysis of the Broadband Noise Scenario in Powerline Networks," in Proc. 4th International Symposium on Power-Line Communications and Its Applications (ISPLC), Limerick, 2000, pp. 849 - 858 .

[14] A. Lasciandare, et al., "Experimental field trials of a utility AMR power line communication system analyzing channel effects and error correction methods," in Proc. IEEE International Symposiumon on Power Line Communications and Its Applications (ISPLC), Pisa, 2007, pp. 144 - 149.

[15] H. Meng, Y. Guan and S. Chen, "Modeling and analysis of noise effects on broadband power-line communications," IEEE Transactions on Power Delivery, vol. 20, no. 2, pp. 630 - 637, 2005.

[16] A. Voglgsang, et al., "Measurement, Characterization and Simulation of Noise on Powerline Channels," in Proc. 4th International Symposium on Power-Line Communications and Its Applications (ISPLC 2000), Limerick, 2000, pp. 139 - 146.

[17] M. Zimmermann and K. Dostert, "Analysis and Modeling of Impulsive Noise in Broad-Band Powerline Communications," IEEE transactions on Electromagnetic Compatibility, vol. 44, no. 1, pp. 249 - 258, 2002.

[18] Y. Fathi, et al., "Practical Issues of Power Line Communication for Automatic Meter Reading Systems," in presented at the 14th International of Middle East Power Systems, Egypt, 2010, pp. 634-640.

[19] D. M. Pozar, "Transmission Line Theory," in Microwave Engineering, New York, JohnWiley \& Sons, Inc., 2011, pp. 48-87.

[20] Enel, "The OPEN meter Consortium," PLC lower layers on b-psk for the Meters And More suite D 5.3.3, 15 June 2011. [Online]. Available: http://www.openmeter.com/files/deliverables/D5.3.3_PLC\%20lower\%201 ayers $\% 20 \mathrm{on} \% 20 \mathrm{~B}$ -

PSK\%20for\%20the\%20Meters\%20and\%20More\%20suite\%20v1.pdf. [Accessed 30 September 2013].

[21] Xania, "Wikibooks-Signal Processing/Digital Filters," 8 April 2013. 
[Online].

Available:

http://en.wikibooks.org/wiki/Signal_Processing/Digital_Filters. [Accessed 14 November 2013].

[22] M. Arzbergerl, K. Dostert, T. Waldeck and M. Zimmermann, "Fundamental Properties of the Low Voltage Power Distribution Grid," in Proc. International Symposium on Power-Line Communications and Its Applications (ISPLC), Germany, 1997, pp. 45 - 50.

[23] A. Nayagam, "Generating the parity check matrix -release 1.0," 27 July 2001. [Online]. Available: http://arun-10.tripod.com/ldpc/generate.html. [Accessed 20 January 2013].

[24] Y. Xiao, "Matlab Central, File exchange, Shortten DVB-S2 LDPC 64800 code into 8100," 22 June 2010. [Online]. Available: http://www.mathworks.com/matlabcentral/fileexchange/27985-shorttendvb-s2-ldpc-64800-code-into-8100. [Accessed 29 January 2014].

[25] D. MacKay and M. Postol, "Weaknesses of Margulis and RamanujanMargulis Low-Density Parity-Check Codes," Electronic Notes in Theoretical Computer Science, vol. 74, p. 8, 2003.

[26] Y. Han and W. Ryan, "LDPC decoder strategies for achieving low error floors," in Proc. Information Theory and Applications Workshop, San Diego, CA, 2008, pp. 277 - 286.

[27] Y. Xiao, "Alternative good LDPC codes for DVB-S2," in Proc. 9th International Conference on Signal Processing (ICSP), Beijing, 2008, pp. 1959 - 1962.

[28] N. Andreadou, C. Assimakopoulos and F. Pavlidou, "Performance Evaluation of LDPC Codes on PLC Channel Compared to Other Coding Schemes," in Proc. IEEE International Symposium on Power Line Communications and Its Applications (ISPLC), Pisa, 2007, pp. 296 - 301.

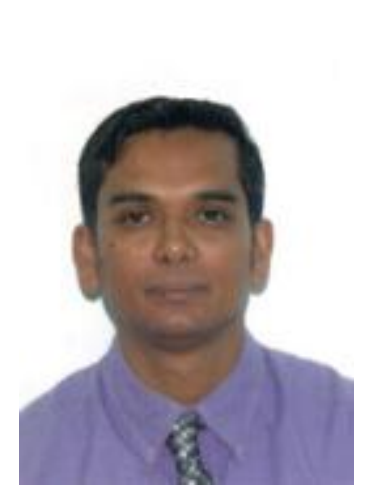

\section{AUTHOR PROFILE}

Dr. Bhimsen Rajkumarsingh holds a degree in Electrical and Electronic Engineering from the University of Mauritius, CAPET degree in Energy systems from IUFM, France and a $\mathrm{PhD}$ in Engineering from the University of Mauritius. Between 1998 and 2010, he held the position of lecturer at the Institute Superieur de Technologie operating under the aegis of the University of Technology. He held the position of Research Officer at the Mauritius Research council in 2008, where he was engaged in national projects regarding energy systems. Currently, he is lecturer at the University of Mauritius in the department of Electrical and Electronic Engineering. His research interests are Energy Systems, Communication Systems and Engineering Education.

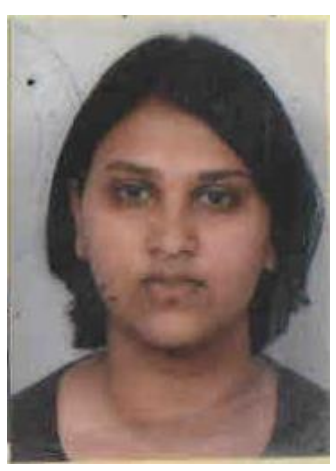

Miss Poonye Namratha Swapna was born on 26 September 1991, in the district of Moka, Mauritius. She is currently studying BEng Electronic and Communication Engineering at the University of Mauritius and working on her thesis. Her present main field of interests involves, Coding theory and Communication Systems for the application in Power-lines. 